D.O.I.: $10.3895 /$ gi.v10i4.1784

\title{
AVALIAÇÃO DA EFICIÊNCIA PORTUÁRIA UTILIZANDO A ANÁLISE ENVOLTÓRIA DE DADOS: UM ESTUDO DOS TERMINAIS DE CONTÊINERES DOS PORTOS DA REGIÃO SUL DO BRASIL
}

\section{PORT EFFICIENCY EVALUATION USING DATA ENVELOPMENT ANALYSIS: A STUDY OF CONTAINER TERMINALS OF SOUTHERN BRAZILIAN PORTS}

\author{
Guilherme Bergmann Borges Vieira ${ }^{1}$; Roberto Birch Gonçalves ${ }^{2}$; Gabriel Sperandio Milan ${ }^{3}$; \\ Alexandre Querino da Rosa ${ }^{4}$ \\ ${ }^{1}$ Universidade de Caxias do Sul - UCS \\ gbbvieir@ucs.br \\ ${ }^{2}$ Universidade de Caxias do Sul - UCS \\ rbgoncal@ucs.br \\ ${ }^{3}$ Universidade de Caxias do Sul - UCS \\ gsmilan@ucs.br \\ ${ }^{4}$ Pontifícia Universidade Católica do Rio Grande do Sul - PUCRS \\ alexandre.querino@yahoo.com.br
}

\begin{abstract}
Resumo
O presente estudo teve como objetivo avaliar comparativamente a eficiência dos terminais de contêineres dos portos da região sul do Brasil. Para tanto, utilizou-se o método da análise envoltória de dados ou Data Envelopment Analysis (DEA). O DEA é indicado para comparar a eficiência relativa de unidades de negócios utilizando diferentes inputs e outputs, ainda que os mesmos estejam em diferentes unidades de medida, uma vez que se trata de uma técnica não paramétrica. Neste estudo, foram considerados como inputs os dados relativos a calado; extensão dos berços, capacidade estática e quantidade de gruas em cada terminal estudado e como outputs os dados relativos à quantidade de navios; movimentação de contêineres e produtividade. A escolha de variáveis de input relacionadas à infraestrutura e variáveis de output associadas à operação tomou como base estudos anteriores sobre eficiência portuária identificados na literatura. A aplicação do DEA mostrou-se útil e os resultados obtidos podem ser usados para orientar o processo de tomada de decisão nos portos estudados.
\end{abstract}

Palavras-chave: avaliação da eficiência; análise envoltória de dados; portos; região sul do Brasil.

\section{Introdução}

Desde tempos remotos, o mar revela-se como espaço de destaque no desenvolvimento da economia mundial, sendo os portos agentes facilitadores para tal desenvolvimento. A história portuária brasileira data de 28/01/1808, data em que foi assinado pelo príncipe regente dom João de Portugal o Decreto de Abertura dos Portos às nações amigas, o qual determinou também que todos os portos fossem administrados pelo Estado (SEP, 2011). 
Passados muitos anos, um cenário de falta de investimentos, aliado ao crescimento do comércio, decorrente da globalização e da abertura de diversas economias, acabou gerando um déficit na infraestrutura portuária, o que vem influenciando a competitividade das organizações, principalmente no mercado externo. Além disso, também se observa um mau uso da infraestrutura existente, o que justifica a importância da realização de estudos sobre eficiência portuária.

A falta de investimentos em infraestrutura no Brasil causou um impacto negativo no desenvolvimento do setor portuário e os portos se tornaram pouco equipados e preparados para atender as novas necessidades do transporte marítimo e prover a necessária agilidade no manuseio de cargas exigida pelos novos padrões internacionais. Atualmente, a grande maioria dos portos brasileiros possui terminais privados que estão investindo para mudar essa realidade e colocar o Brasil em um novo patamar de eficiência e qualidade dos serviços.

Nesse sentido, devem-se ponderar alguns fatores relacionados com a eficiência portuária para identificar comparativamente os portos mais eficientes e orientar ações de melhoria no setor. Atualmente, o ambiente portuário da Região Sul do Brasil é composto por sete portos: i) Paranaguá; ii) Itapoá; iii) São Francisco do Sul; iv) Itajaí; v) Navegantes; vi) Imbituba; e vi) Rio Grande. Alguns desses portos estão entre os mais importantes do Brasil na movimentação de cargas, tanto de exportação quanto de importação, e são de relevante importância para o desenvolvimento regional e nacional.

Dados esse contexto, o presente trabalho teve como objetivo geral analisar a eficiência dos portos da região sul do Brasil através do método da Análise Envoltória de Dados (DEA). Para tanto, estabeleceu-se como objetivos específicos: i) caracterizar os portos estudados; ii) selecionar informações para a correta escolha de variáveis de input e output a serem utilizadas no DEA; iii) analisar a eficiência dos portos com base no DEA; iv) avaliar os resultados obtidos, indicando um direcionamento para o aumento da eficiência dos portos estudados.

A justificativa da pesquisa baseia-se em três elementos principais: i) para o ambiente portuário como base e parecer independente sobre a eficiência portuária regional; ii) para o ambiente externo, pois poderá contribuir para o desenvolvimento e tomada de decisões tanto em nível político como no âmbito privado para organizações focadas em operações que envolvam ou se utilizem do universo portuário; e iii) para o meio acadêmico, uma vez que o estudo poderá servir de referência para pesquisas futuras.

\section{Eficiência, eficácia e produtividade: principais conceitos}

Em um mundo cada vez mais focado em resultados, não se fala somente em produzir, mas sim em produzir bem. Nesse contexto, devem ser considerados os conceitos de produtividade, eficiência e eficácia. 
De acordo com Coelli, Rao e Battese (1999), a produtividade é a razão entre dois fatores: i) o que foi produzido; ii) o que foi gasto. Conforme relatório da CEPAL (2006), a busca pela produtividade pode trazer muitas vantagens como: i) a otimização de recursos; ii) a identificação de unidades de negócio mais produtivas; iii) a definição de estratégias a fím de aumentar a produtividade; iv) o redirecionamento de uma unidade de negócio quando esta está ineficiente; e v) a definição do nível de investimento exigido para incrementar a produção. Sabe-se que as vantagens são inúmeras quando se trabalha na busca pela produtividade, porém pode-se lidar com alguns limitadores. Por exemplo, dentro de uma indústria, mesmo adotando-se uma série de ações para aumentar a produtividade, pode-se não obter um adequado nível de satisfação do cliente. Portanto, deve-se considerar também a eficácia ou grau de atendimento às especificações internas ou expectativas externas. A eficácia está ligada ao que é produzido, não levando em conta os inputs fatores que contribuem para produção do produto e/ou serviço (MELLO et al, 2005). Assim sendo, pode-se dizer que a eficácia é um fator importante para a análise do desempenho, mas, avaliada isoladamente, não é o mais adequado, pois desconsidera os dados de entrada (inputs).

É nesse ponto que entra a eficiência. Segundo Pearson (1993), a eficiência é a medida de proximidade entre o que foi produzido e a quantidade de referência. Rios e Maçada (2006), por sua vez, considerando a lógica da análise envoltória de dados, afirmam que a eficiência deve ser a comparação entre os produtos (outputs) observados e os máximos produtos alcançáveis para os recursos (inputs) utilizados. De qualquer forma, seja qual for a abordagem utilizada, a eficiência é um elemento importante para orientar melhorias que permitam a redução de custos e o aumento da competitividade. E a análise envoltória de dados é uma ferramenta útil para a análise da eficiência relativa, dado um grupo de unidades produtivas.

Os portos fazem parte de um sistema de transporte, com funções de deslocar pessoas e mercadorias. Collyer (2008) define porto como fronteira nacional aberta, entreposto dinâmico de mercadorias, em que se realizam atividades (aduaneiras, alfandegárias, comerciais, sanitárias, tributárias, imigratórias etc.). É o portão de entrada e saída de riquezas, local de abrigo para embarcações, fonte de suprimentos das atividades offshore, ponto estratégico para a segurança das nações e, sobretudo, o mais importante elo da cadeia logística global.

Em relação aos portos, a eficiência pode ser notada considerando-se aspectos diversos, mas necessariamente interligados entre si, objetivando melhorar a performance. Sob o ponto de vista da distribuição de cargas globais, a eficiência é melhorada pelo tanto pela adequação da infraestrutura quanto pelo aprimoramento da organização e do gerenciamento interno. Nesse sentido, Notteboom e Rodrigue (2005) abordam a conteinerização, a SCM e a configuração do interior do porto como aspectos fundamentais que contribuem com a eficiência. Além disso, é importante considerar que a eficiência portuária se reflete na capacidade competitiva de uma região e, por extensão, de um país, 
sendo necessárias políticas governamentais compatíveis e uma forte atenção empresarial para que isso aconteça.

\section{Análise envoltória de dados (DEA): aplicações no setor portuário}

O método da Análise Envoltória de Dados (DEA) baseia-se em programação matemática e possibilita análises comparativas entre várias unidades produtivas, denominadas Decision Making Units (DMUs), inclusive prestadoras de serviços. Seu surgimento caracterizou-se como uma evolução dos métodos tradicionais de medição da eficiência das unidades produtivas. Cabe salientar, entretanto, que o DEA vai além do cálculo da eficiência, permitindo a identificação da ineficiência e a fundamentação para o desenvolvimento de planos de ações aplicáveis às DMUs ineficientes.

Segundo Charnes, Cooper e Rhodes (1978), formuladores e desenvolvedores do DEA, este método baseia-se em uma programação matemática onde a medida da eficiência é resultante da razão da soma ponderada dos dados de saída (outputs) pelos de entrada (inputs). Conforme Yun, Nakayama e Tanino (2004), o DEA possui algumas características relevantes, tais como: i) pode ser aplicado para medir diversos inputs e diversos outputs sem que sejam determinados pesos aos mesmos; ii) pode ser utilizado na medição da eficiência a partir de dados empíricos, mesmo que não se conheça a função produção; e iii) os tomadores de decisão podem incorporar suas preferências ao modelo DEA. Outros autores defendem que o DEA serve para apoio na tomada de decisão multicritério, procurando modelar a complexidade da realidade (LINS e ANGULO-MEZA, 2000). Cook e Zhu (2005) definem DEA como modelo para avaliar o desempenho de entidades onde se podem converter as várias entradas (inputs) para várias saídas (outputs). Thanassoulis, Boussofiane e Dyson (1996) visualizam o DEA como uma abordagem não paramétrica para estimar o máximo de produção para os dados de entrada (inputs) ou valores mínimos necessários de entrada para uma certa produção (outputs). O DEA possibilita a análise comparativa de processos em escalas diferentes com o auxílio da fronteira de produção, possibilitando a hierarquização dos processos segundo um critério de desempenho previamente definido (NOVAES, 1996).

No segmento portuário, foram realizados diversos estudos utilizando o DEA. MartínezBudría et al (1999) analisaram a eficiência de 26 portos espanhóis utilizando o DEA. Pela complexidade e variedade de tamanho os portos, os mesmos foram divididos em três grupos a fim de que fosse possível alcançar resultados conclusivos.

Tongzon (2001), por sua vez, mediu a performance de quatro portos australianos, além de outros doze portos internacionais, em 1996. Seu estudo demonstrou que a técnica DEA é útil e adequada para a medição da eficiência em terminais de contêineres. 
Outros países com portos e terminais de contêineres reconhecidos mundialmente também foram estudados. Estados Unidos, Inglaterra, Austrália e outros foram alvos das análises de eficiência de Valentine e Gray (2001). Segmentando esses portos entre privados e públicos, a pesquisa buscou verificar se algum tipo específico de administração e estrutura organizacional leva os portos a serem mais eficientes. Na ocasião, foram estudados 21 terminais de contêineres que faziam parte da lista dos 100 melhores conforme a revista Cargo Systems de 1999. Foram escolhidos como inputs o tamanho dos berços de atracação e o total de investimentos e os outputs foram o número total de contêineres e a quantidade total de toneladas movimentadas. O resultado fez com que o autor sugerisse uma quantidade maior de inputs na equação, pois outros dados seriam relevantes a serem considerados no estudo, uma vez que os dados apresentados mostraram apenas que a estrutura organizacional poderia ser incorporada em um modelo conceitual portuário.

De forma mais abrangente, pode-se definir o DEA como sendo um método não paramétrico que serve para avaliar a eficiência comparativa de entidades (DMUs) responsáveis pela realização de operações semelhantes com múltiplos dados de entrada (inputs) e saída (outputs) com o objetivo de modelar a realidade atual.

Para a compreensão da análise DEA, é importante ter definidos os fatores de escala e a chamada fronteira de produção ligada à eficiência. Os fatores de escala são as respostas da produção ao aumento da quantidade de recursos (inputs), sendo que podem ser divididas em constantes e variáveis. Quando a quantidade de recursos (inputs) aumenta ou diminui proporcionalmente à quantidade de produtos (outputs), esses fatores são denominados constantes. Já os fatores de escala variáveis podem ser crescentes ou decrescentes. Os fatores de escala crescentes ocorrem quando o aumento dos recursos (inputs) gera um aumento mais que proporcional dos produtos (outputs). Quando esses acréscimos de recursos (inputs) geram um acréscimo menos que proporcional de produtos (outputs) chama-se de fator de escala decrescente, conforme Sousa Junior (2010).

O método DEA pode ser analisado utilizando dois modelos principais: i) Modelo CCR, criado por Charnes, Cooper e Rhodes (1978), que trabalha com um constante retorno à escala de produção, ou seja, alterações promovidas nos recursos (inputs) provocam alterações proporcionais nos produtos (outputs); ii) Modelo BCC, criado por Banker, Charnes e Cooper (1984), que prevê retornos variáveis, não levando em consideração a proporcionalidade entre os recursos (inputs) e os produtos (outputs). Portanto, o modelo BCC considera a possibilidade de rendimentos crescentes ou decrescentes de escala na fronteira eficiente, obrigando que essa seja convexa.

Como dito anteriormente, os estudos sobre a eficiência portuária com aplicação do DEA podem utilizar diferentes modelos (BCC ou CCR, orientados a inputs ou outputs) e variados dados de entrada e saída. A Figura 1 apresenta alguns exemplos recentes de aplicações do DEA no setor portuário. 
Observa-se na Figura 1 a existência de diversos estudos sobre eficiência portuária utilizando o DEA, desenvolvidos em diversas regiões, inclusive no Brasil, e considerando variados inputs e outputs. Entre as variáveis de output consideradas, destacam-se as relacionadas com a movimentação de cargas (em toneladas ou TEUs - Twenty Feet Equivalent Units, unidades de contêineres de $20^{\prime}$ ), enquanto que as variáveis de input estão relacionadas com a infraestrutura portuária, destacando-se a área dos terminais, o comprimento do cais e o número de guindastes como principais variáveis.

Figura 1 - Exemplos de estudos utilizando o DEA no setor portuário

\begin{tabular}{|c|c|c|c|}
\hline Autores & Inputs & Outputs & Amostra \\
\hline $\begin{array}{l}\text { Hung, Lu e Wang } \\
\qquad(2010)\end{array}$ & $\begin{array}{c}\text { Número de berços; Área do terminal em m²; Número } \\
\text { de guindastes do cais (ship-shore container gantry); } \\
\text { Comprimento do cais em metros. }\end{array}$ & $\begin{array}{l}\text { Movimentação anual } \\
\text { em TEUs }\end{array}$ & 31 portos da Ásia \\
\hline $\begin{array}{l}\text { Sharma e Yu } \\
\quad(2010)\end{array}$ & $\begin{array}{c}\text { Área do terminal em } \mathrm{m}^{2} \text {; Comprimento do cais em } \\
\text { metros; Número de guindastes (cranes); Número de } \\
\text { guindastes de transferência (transfer cranes); } \\
\text { Número de empilhadeiras de alcance (reach stacker); } \\
\text { Número de equipamentos de movimentação de } \\
\text { contêineres (straddle carriers). }\end{array}$ & $\begin{array}{l}\text { Movimentação anual } \\
\text { em TEUs }\end{array}$ & $\begin{array}{l}70 \text { portos de } \\
\text { contêineres }\end{array}$ \\
\hline $\begin{array}{l}\text { Lozano, Villa e } \\
\text { Canca (2011) }\end{array}$ & $\begin{array}{l}\text { Área do terminal em } \mathrm{m}^{2} \text {; Comprimento do cais em } \\
\text { metros; Número de guindastes (cranes); Número de } \\
\text { rebocadores (tugs). }\end{array}$ & $\begin{array}{l}\text { Movimentação anual } \\
\text { em TEUs; Tráfego de } \\
\text { cargas em toneladas/ } \\
\text { ano; Número de } \\
\text { navios por ano. }\end{array}$ & Portos espanhóis \\
\hline Barros (2012) & $\begin{array}{l}\text { Área total do terminal em } \mathrm{m}^{2} \text {; Comprimento do cais } \\
\text { em metros; Número de trabalhadores. }\end{array}$ & $\begin{array}{l}\text { Movimentação anual } \\
\text { em TEUs; Granéis } \\
\text { sólidos em toneladas; } \\
\text { Granéis líquidos em } \\
\text { toneladas; Número de } \\
\text { dias para a } \\
\text { movimentação da } \\
\text { carga. }\end{array}$ & Portos africanos \\
\hline $\begin{array}{l}\text { Niavis e Tsekeris } \\
\text { (2012) }\end{array}$ & $\begin{array}{l}\text { Comprimento do cais em metros; Número de berços; } \\
\text { Número de guindastes. }\end{array}$ & $\begin{array}{l}\text { Movimentação anual } \\
\text { em TEUs }\end{array}$ & $\begin{array}{l}30 \text { portos do } \\
\text { sudeste da Europa }\end{array}$ \\
\hline Wanke (2013) & $\begin{array}{l}\text { Número de berços; Área do armazém em m²; Área } \\
\text { do páito em } \mathrm{m}^{2} .\end{array}$ & $\begin{array}{l}\text { Movimentação anual } \\
\text { em TEUs; } \\
\text { Rendimento anual dos } \\
\text { granéis sólidos em } \\
\text { toneladas. }\end{array}$ & $\begin{array}{l}27 \text { portos } \\
\text { brasileiros }\end{array}$ \\
\hline Bichou (2013) & $\begin{array}{l}\text { Área total do terminal em } \mathrm{m}^{2} \text {; Comprimento do cais } \\
\text { em metros; Número de portões (gates); Número de } \\
\text { guindastes de cais e pátio, equipamentos de } \\
\text { movimentação de carga, esteiras, caminhões, tratores } \\
\text { e outros veículos internos. }\end{array}$ & $\begin{array}{l}\text { Movimentação anual } \\
\text { em TEUs }\end{array}$ & $\begin{array}{l}420 \text { terminais de } \\
\text { contêineres }\end{array}$ \\
\hline $\begin{array}{l}\text { Yuen, Zhang e } \\
\text { Cheung (2013) }\end{array}$ & $\begin{array}{l}\text { Área do terminal em m²; Comprimento do cais em } \\
\text { metros; Número de berços; Número de guindastes de } \\
\text { cais; Número de guindastes de pátio. }\end{array}$ & $\begin{array}{l}\text { Movimentação anual } \\
\text { em TEUs }\end{array}$ & Portos chineses \\
\hline $\begin{array}{l}\text { Trujillo, González } \\
\text { e Jiménez (2013) }\end{array}$ & $\begin{array}{c}\text { Área total do terminal em } \mathrm{m}^{2} ; \text { Berços em metros; } \\
\text { Número de guindastes. }\end{array}$ & $\begin{array}{l}\text { Movimentação anual } \\
\text { em TEUs }\end{array}$ & Portos africanos \\
\hline
\end{tabular}




\section{Procedimentos metodológicos}

Este capítulo descreve os procedimentos metodológicos utilizados neste trabalho, abordando os seguintes aspectos: amostra; modelo de DEA utilizado; variáveis consideradas e plano de coleta de dados. No presente estudo, foram considerados como amostra sete terminais de contêineres de sete portos da região sul do Brasil: Paranaguá, Itapoá, São Francisco do Sul, Itajaí, Navegantes, Imbituba e Rio Grande.

Quanto ao modelo de DEA utilizado, considerou-se o CCR, o qual é o modelo DEA mais conhecido e utilizado (SOUSA JUNIOR, 2010). O CCR é um modelo utilizado quando existe uma relação entre inputs e outputs com escalas constantes. Foi o primeiro modelo DEA desenvolvido e passou a ser chamado assim depois que Charnes, Cooper e Rodhes o apresentaram em 1978. O modelo CCR pode ser estruturado supondo n DMUs utilizando i inputs para produzir o outputs. O CCR pode ser orientado a input ou a output. No presente caso, foi considerada a orientação a inputs.

A seleção de variáveis deu-se para um único tipo de carga, o contêiner. Foram selecionadas variáveis de input relacionadas à infraestrutura e variáveis de output associadas à operação. As seguintes variáveis foram consideradas como inputs: i) calado (m); ii) extensão do berço (m); iii) capacidade estática (em TEUs); e iv) número de guindastes (portainers). Já os outputs considerados foram os seguintes: i) navios (quantidade); ii) movimentação (em TEUs); iii) Produtividade no cais (unid/h). Para a seleção das variáveis, considerou-se como referência a literatura da área, especialmente o estudo de Sousa Junior (2010). Foi considerado esse estudo por ter sido desenvolvido no Brasil (região nordeste), de forma metódica, e por refletir significativamente as recomendações encontradas na literatura.

No que diz respeito ao plano de coleta de dados, trabalhou-se exclusivamente com dados secundários, disponíveis no sistema Desempenho Portuário da Agência Nacional de Transportes Aquaviários (ANTAQ) e complementados por informações obtidas junto aos portos estudados.

\section{Resultados}

O presente capítulo apresenta os resultados da pesquisa, sendo apresentados os resultados da análise envoltória de dados (DEA). Os portos e terminais que operam contêineres na região sul do Brasil e foram considerados no estudo são os seguintes: i) TCP - Terminal de Contêineres de Paranaguá, no Porto de Paranaguá/PR; ii) Terminal de Contêineres de Itapoá, no Porto de Itapoá/SC; iii) TESC - Terminal Santa Catarina, no Porto de São Francisco do Sul/SC; iv) APM Terminal Itajaí, no Porto de Itajaí/SC; v) Portonave, no Porto de Navegantes/SC; vi) TECON Imbituba, no Porto de Imbituba/SC; e vii) TECON Rio Grande, no Porto de Rio Grande/RS. Para a análise envoltória de dados, as variáveis consideradas como inputs foram as seguintes: i) calado 
(m); ii) extensão do berço (m); iii) capacidade estática (TEUs), e iv) número de guindastes (portainers). Já as variáveis consideradas como outputs foram: i) navios (quantidade); ii) movimentação (TEUs); e iii) produtividade no cais (unid/h). Os dados coletados para cada porto em cada variável são apresentados na Tabela 1 .

Conforme se observa na Tabela 1, os terminais TCP e TECON Rio Grande dos portos de Paranaguá e Rio Grande, respectivamente, apresentaram a maior quantidade de contêineres movimentados, seguidos dos terminais Portonave (Navegantes) e APM (Itajaí). Quanto à produtividade $(\mathrm{mph})$ dos portainers, percebe-se que o terminal Itajaí registra a maior produtividade (77 mph), sendo seguido por Navegantes $(60 \mathrm{mph})$ e Itapoá (54 mph). Observa-se que o TECON Rio Grande apresentou uma maior disponibilidade de equipamentos para movimentação de contêineres (portainers), mas não conseguiu registrar uma produtividade superior aos seus concorrentes Itajaí, Navegantes e Itapoá.

Tabela 1 - Dados de input e output para cada porto estudado

\begin{tabular}{c|c|cccc|ccc}
\hline Porto & Terminal & \multicolumn{4}{|c|}{ Inputs } & \multicolumn{3}{c}{ Outputs } \\
& & $\begin{array}{c}\text { Calado } \\
(\mathrm{m})\end{array}$ & $\begin{array}{c}\text { Berços } \\
(\mathrm{m})\end{array}$ & $\begin{array}{c}\text { Capac. } \\
\text { (TEUs) }\end{array}$ & $\begin{array}{c}\text { Portainers } \\
\text { (unid) }\end{array}$ & $\begin{array}{c}\text { Navios } \\
\text { (un) }\end{array}$ & $\begin{array}{c}\text { Mov. } \\
\text { (TEUs) }\end{array}$ & $\begin{array}{c}\text { Prod. } \\
(\mathrm{mph})\end{array}$ \\
\hline Paranaguá & TCP & 12,3 & 564 & 28.000 & 4 & 830 & 396.933 & 35 \\
Itapoá & Itapoá & 16 & 630 & 10.000 & 4 & 112 & 23.928 & 54 \\
SFco do Sul & TESC & 12 & 603 & 3.000 & 1 & 318 & 120.750 & 40 \\
Itajaí & APM & 11,4 & 535 & 14.000 & 2 & 434 & 258.262 & 77 \\
Navegantes & Portonave & 11 & 900 & 20.000 & 3 & 619 & 350.529 & 60 \\
Imbituba & Tecon Imbituba & 11 & 660 & 7.444 & 2 & 186 & 11.537 & 40 \\
Rio Grande & Tecon Rio Grande & 14,5 & 900 & 30.000 & 6 & 854 & 382.933 & 49 \\
\hline
\end{tabular}

Fonte: Dados da ANTAQ e dos portos estudados

A Tabela 2 apresenta os resultados da análise envoltória de dados. Percebe-se que, na média, os portos da região sul apresentam um elevado grau de eficiência (92,9\%). Paranaguá, São Francisco do Sul, Itajaí e Navegantes são os portos mais eficientes (100\%). O porto de Rio Grande aparece a seguir, com uma eficiência relativa de 93,9\%. Já os portos de Itapoá e Imbituba apresentam uma eficiência menor, de $80,7 \%$ e $75,4 \%$, respectivamente.

\begin{tabular}{ll}
\multicolumn{2}{c}{ Tabela 2 - Eficiência relativa dos portos } \\
\hline Porto & Eficiência \\
\hline Paranaguá & 1,000 \\
Itapoá & 0,807 \\
São Francisco do Sul & 1,000 \\
Itajaí & 1,000 \\
Navegantes & 1,000 \\
Imbituba & 0,754 \\
Rio Grande & 0,939 \\
\hline Média & 0,929 \\
\hline
\end{tabular}


No que se refere às referências de eficiência (peers), percebe-se que o porto de Itajaí é referência para Itapoá e Imbituba, enquanto o porto de São Francisco do Sul é referência para Itapoá, Imbituba e Rio Grande (Tabela 3).

Tabela 3 - Lista de referências

\begin{tabular}{ll}
\hline Porto & Referências (peers) \\
\hline Paranaguá & Paranaguá \\
Itapoá & Itajaí e São Francisco do Sul \\
São Francisco do Sul & São Francisco do Sul \\
Itajaí & Itajaí \\
Navegantes & Navegantes \\
Imbituba & Itajaí e São Francisco do Sul \\
Rio Grande & São Francisco do Sul, Paranaguá e Navegantes \\
\hline Fonte: Autoria própria &
\end{tabular}

Quanto às metas de desempenho de outputs de todos os portos, percebe-se na Tabela 4 que, considerados os inputs existentes, os portos de Itapoá e Imbituba deveriam apresentar um maior número de navios atendidos e também uma maior movimentação de contêineres. Já o porto de Rio Grande deveria apenas aumentar ligeiramente a movimentação de contêineres.

\begin{tabular}{l|cc|cc|cc}
\multicolumn{7}{c}{ Tabela 4 - Resumo das metas de outputs } \\
\hline Porto & \multicolumn{2}{|c|}{ Navios (un) } & \multicolumn{2}{c}{ Movimentação (TEUs) } & \multicolumn{2}{c}{ Produtividade (mph) } \\
& Real & Meta & Real & Meta & Real & Meta \\
\hline Paranaguá & 830 & 830 & 396.933 & 396.933 & 35 & 35 \\
Itapoá & 112 & 342 & 23.928 & 175.627 & 54 & 54 \\
São Francisco do Sul & 318 & 318 & 120.750 & 120.750 & 40 & 40 \\
Itajaí & 434 & 434 & 258.262 & 258.262 & 77 & 77 \\
Navegantes & 619 & 619 & 350.529 & 350.529 & 60 & 60 \\
Imbituba & 186 & 261 & 11.537 & 128.940 & 40 & 40 \\
Rio Grande & 854 & 854 & 382.933 & 426.046 & 49 & 49 \\
\hline
\end{tabular}

Fonte: Autoria própria

Quanto às metas de desempenho de inputs de cada porto, observa-se na Tabela 5 que os portos de Itapoá, Imbituba e Rio Grande apresentam uma folga na extensão de berços e na capacidade estática, considerando os dados de saída (outputs) existentes.

Tabela 5 - Resumo das metas de inputs

\begin{tabular}{l|cc|cc|cc|cc}
\hline \multirow{2}{*}{ Porto } & \multicolumn{2}{|c|}{ Calado $(\mathrm{m})$} & \multicolumn{2}{c|}{ Berço $(\mathrm{m})$} & \multicolumn{2}{c|}{ Capac. (TEUs) } & \multicolumn{2}{c}{ Portainers (un) } \\
& Real & Meta & Real & Meta & Real & Meta & Real & Meta \\
\hline Paranaguá & 12,3 & 12,3 & 564 & 564 & 28.000 & 28.000 & 4 & 4 \\
Itapoá & 16,0 & 10,5 & 630 & 508 & 10.000 & 8.068 & 4 & 1 \\
São Francisco do Sul & 12,0 & 12,0 & 603 & 603 & 3.000 & 3.000 & 1 & 1 \\
Itajaí & 11,4 & 11,4 & 535 & 535 & 14.000 & 14.000 & 2 & 2 \\
Navegantes & 11,0 & 11,0 & 900 & 900 & 20.000 & 20.000 & 3 & 3 \\
Imbituba & 11,0 & 8,3 & 660 & 404 & 7.444 & 5.609 & 2 & 1 \\
Rio Grande & 14,5 & 13,6 & 900 & 764 & 30.000 & 28.163 & 6 & 4 \\
\hline \multicolumn{19}{r}{ Fonte: Autoria própria }
\end{tabular}

O mesmo foi observado para o input calado, mas, dadas as características do setor e o aumento no tamanho dos navios, poderia não ser viável em termos operacionais a redução do 
mesmo. No que se refere a essa folga observada, deve-se considerar, ainda, a necessidade de antecipação dos investimentos em infraestrutura portuária, associada a um planejamento de longo prazo voltado a uma movimentação futura projetada, o que também é característico do setor. Portanto, certo grau de folga pode ser considerado normal ou até mesmo desejável nesse setor.

\title{
5. Conclusão
}

O trabalho descreveu as principais características dos portos da região sul do Brasil e da Análise Envoltória de Dados. Com a seleção das variáveis utilizadas no modelo e a coleta de dados dos portos, foi elaborado um modelo DEA CCR orientado a inputs e foram apresentados os índices de eficiência, as referências (peers) e as metas de output e input de cada porto.

Os resultados indicam que os portos de Paranaguá, São Francisco do Sul, Itajaí e Navegantes são os mais eficientes, enquanto que Itapoá, Imbituba e Rio Grande apresentam uma eficiência relativa menor. Os portos menos eficientes foram os de Imbituba e Itapoá. No entanto, devem-se ressaltar algumas particularidades desses dois portos. Em Itapoá, que tem uma infraestrutura moderna, devido ao investimento ter sido concretizado recentemente, isso ainda não se refletiu em melhoria de desempenho ou eficiência portuária. O mesmo pode-se dizer de Imbituba, que recebeu recentemente investimentos em novos equipamentos e berços, mas tais investimentos ainda não geraram um aumento proporcional na movimentação de cargas.

Os resultados encontrados comprovam a aplicabilidade da análise envoltória de dados, reforçam a importância de analisar a eficiência portuária e sugerem a necessidade de um contínuo acompanhamento da mesma na região estudada.

\begin{abstract}
The present study aimed at comparing the efficiency of container ports in southern Brazil. For this purpose, the method of Data Envelopment Analysis (DEA) was used. The DEA is suitable for comparing the efficiency of business units using different inputs and outputs, even if they are in different units of measurement, since it is a non-parametric technique. In this study, it was considered as inputs draught; berth extension, static capacity and number of cranes at each terminal and as outputs number of ships; container throughput and productivity. The choice of input variables related to infrastructure and output variables associated with the operation was based on previous studies on port efficiency identified in the literature. The application of DEA was useful and the results obtained allow guiding the process of decision making in the ports studied.
\end{abstract}

Key-words: efficiency evaluation; data envelopment analysis; ports; southern Brazil.

\section{Referências}

BANKER, R. D.; CHARNES, A.; COOPER, W. W. Some models for estimating technical and scale inefficiencies in data envelopment analysis. Management Science, v.30, n. 9, p. 1078-1092, 1984. crossref

BARROS, C. P. Decomposing Growth in Portuguese Seaports: A Frontier Cost Approach. Maritime Economics \& Logistics, v. 7, n. 1, p. 297-315, 2005. crossref 
BARROS, C. P. Productivity Assessment of African Seaports. African Development Review, v. 24, n. 1, p. 67-78, 2012. cross ref

BICHOU, K. An empirical study of the impacts of operating and market conditions on container- port efficiency and benchmarking. Research in Transportation Economics, v. 42, n. 1, p. 28-37, 2013. crossref

BRASIL, Secretaria Especial dos Portos da Presidência da República (SEP/PR). Site Institucional. Disponível em: <http://www.portosdobrasil.gov.br/>. Acesso em: 07 nov. 2011.

CEPAL - Comissão Econômica para América Latina e Caribe. Indicadores de productividad para la industria portuaria: Aplicación en América Latina y el Caribe, 2006.

CHARNES, A.; COOPER, W. W.; RODHES, E. Measuring the efficiency of decision marking units. European Journal of Operational Research, v. 2, n. 6, p. 429-444, 1978. crossref

COELLI, T.; RAO, D. S. P.; BATTESE, G. E. An introduction of efficiency and productivity analysis. Boston: Kluwer Academic Publishers, 1999

COLLYER, W. O. Lei dos Portos: O Conselho de Autoridade Portuária e a Busca da Eficiência. São Paulo: Lex Editora, 2008.

COOK, W. D., ZHU, J. Modeling Performance Measurement: Applications and Implementation Issues in DEA. Springer: New York, 2005.

LINS, M. P E.; ANGULO-MEZA, L. Análise Envoltória de Dados e Perspectivas de Integração no Ambiente de Apoio à Decisão. Rio de Janeiro: Editora da COPPE/UFRJ, 2000.

HUNG, S. W; LU, W. M; WANG, T. P. Benchmarking the operating efficiency of Asia container ports. European Journal of Operational Research, v. 203, n. 3, p.706-713, 2010. crossref

LOZANO, S; VILLA, G; CANCA, D. Application of centralised DEA approach to capital budgeting in Spanish ports. Computers \& Industrial Engineering, v. 60, n. 1, p. 455-465, 2011. cross ref

MARTÍNEZ-BUDRÍA, E.; DÍAZ-ARMAS, R.; NAVARRO-IBAÑEZ, M.; RAVELOMESA, T. A Study of the Efficiency of Spanish Port Authorities using Data Envelopment Analysis. International Journal of Transport Economics, v. 26, n. 2, p. 237-253, 1999.

NIAVIS, S; TSEKERIS, T. Ranking and causes of inefficiency of container seaports in South-Eastern Europe. Eur. Transp. Res. Rev., v. 4, n. 1, p. 235-244, 2012. crossref

NOTTEBOOM, T.; RODRIGUE, J. P. Port regionalization: Towards a new phase in port development. Maritime Policy and Management, v. 32, n. 3, p. 87-105, p. 297-313, 2005.

NOVAES, A. G. N. Avaliação da produtividade de serviços de transportes através da análise envoltória de dados. Anais do X ANPET - Brasília-DF, vol. 2, p. 577-587, 1986.

PEARSON, K. Data Envelopment Analysis: an explanation. Working Paper n. 83, Bureau of Industry Economics, 93. Canberra, 1993.

RIOS, L. R.; MAÇADA, A. C. G. Analysing the relative efficiency of containers terminals of Mercosul using DEA. Maritime Economics and Logistics, v. 8, n. 4, p. 331-346, 2006. crossref

SHARMA, M. J.; YU, S. J. (2010) Benchmark optimization and attribute identification for improvement. European Journal of Operational Research, v. 201, n. 2, p.568-580, 2010. crossref

MELLO, J. C. C. B. S.; ÂNGULO-MEZA, L.; GOMES, E. G.; BIONDI NETO, L. Curso de análise envoltória de dados. In: XXXVII Simpósio Brasileiro de Pesquisa Operacional (SBPO). Anais... Gramado-RS, 2005.

SOUSA JUNIOR, J. N. C. Avaliação da Eficiência dos Portos utilizando Análise Envoltória de Dados: estudo de caso dos portos da região nordeste do Brasil. Dissertação de Mestrado. Programa de Mestrado em Engenharia de Transportes, Universidade Federal do Ceará, 2010. 
THANASSOULIS, E.; BOUSSOFIANE, A.; Dyson, R. G. A comparison of data envelopment analysis and ratios as tools for performance assessment. The International Journal of Management Science, v. 24, n. 3, p. $229-244$, 1996.

TONGZON, J. Efficiency measurement of selected Australian and other international ports using data envelopment analysis. Transportation Research Part A: Policy and Practice, v. 35, n. 2, p. 113-128, 2001. crossref

TRUJILLO, L; GONZÁLEZ, M. M; JIMÉNEZ, J. L. An overview on the reform process of African ports. Utilities Policy, v. 25, n. 1, p.12-22, 2013. crossref

VALENTINE, V. F.; GRAY, R. The Measurement of Port Efficiency using Data Envelopment Analysis. Proceedings of the 9th World Conference on Transport Research, 2001.

WANKE, P. F. Physical infrastructure and shipment consolidation efficiency drivers in Brazilian ports: A two-stage network- DEA approach. Transport Policy, v. 29, n. 1, p. 145-153, 2013. crossref

YUEN, A. C. L; ZHANG, A; CHEUNG, W. (2013) Foreign participation and competition: A way to improve the container port efficiency in China? Transportation Research Part A, v. 49, n.1, p. 220-231. crossref

YUN, Y. B.; NAKAYAMA, H.; TANINO, T. (2004) A generalized model for data envelopment analysis. European Journal of Operational Research, v. 157, n.1, p. 87-105. cross ref

\section{Anexo}

Results from DEAP Version 2.1

Instruction file $=$ por3-ins.txt

Data file = por3-dta.txt

Input orientated DEA

Scale assumption: CRS

Slacks calculated using multi-stage method

EFFICIENCY SUMMARY:

$\begin{array}{cc}\text { firm } & \text { te } \\ 1 & 1.000 \\ 2 & 0.807 \\ 3 & 1.000 \\ 4 & 1.000 \\ 5 & 1.000 \\ 6 & 0.754 \\ 7 & 0.939 \\ \text { mean } & 0.928\end{array}$

\section{SUMMARY OF OUTPUT SLACKS:}

$\begin{array}{clll}\text { firm output: } 1 & 2 & 3 \\ 1 & 0.000 & 0.000 & 0.000 \\ 2 & 230.261 & 151698.530 & 0.000 \\ 3 & 0.000 & 0.000 & 0.000 \\ 4 & 0.000 & 0.000 & 0.000 \\ 5 & 0.000 & 0.000 & 0.000 \\ 6 & 75.488 & 117402.965 & 0.000 \\ 7 & 0.000 & 43113.155 & 0.000 \\ \text { mean } & 43.678 & 44602.093 & 0.000\end{array}$


SUMMARY OF INPUT SLACKS:

$\begin{array}{cllll}\text { firm input: } & 1 & 2 & 3 & 4 \\ 1 & 0.000 & 0.000 & 0.000 & 0.000 \\ 2 & 2.426 & 0.000 & 0.000 & 1.841 \\ 3 & 0.000 & 0.000 & 0.000 & 0.000 \\ 4 & 0.000 & 0.000 & 0.000 & 0.000 \\ 5 & 0.000 & 0.000 & 0.000 & 0.000 \\ 6 & 0.000 & 92.820 & 0.000 & 0.483 \\ 7 & 0.000 & 80.439 & 0.000 & 1.534 \\ \text { mean } & 0.347 & 24.751 & 0.000 & 0.551\end{array}$

SUMMARY OF PEERS:

$\begin{array}{clll}\text { firm } & \text { peers: } & \\ 1 & 1 & & \\ 2 & 4 & 3 & \\ 3 & 3 & & \\ 4 & 4 & & \\ 5 & 5 & & \\ 6 & 4 & 3 & \\ 7 & 3 & 1 & 5\end{array}$

SUMMARY OF PEER WEIGHTS:

(in same order as above)

Firm peer weights:

$1 \quad 1.000$

20.4890 .410

$3 \quad 1.000$

$4 \quad 1.000$

$5 \quad 1.000$

$6 \quad 0.3170 .389$

$7 \quad 0.0440 .7520 .349$

PEER COUNT SUMMARY:

(i.e., no. times each firm is a peer for another)

firm peer count:

$\begin{array}{ll}1 & 1 \\ 2 & 0 \\ 3 & 3 \\ 4 & 2 \\ 5 & 1 \\ 6 & 0 \\ 7 & 0\end{array}$


SUMMARY OF OUTPUT TARGETS:

$\begin{array}{clll}\text { firm output: } & 1 & 2 & 3 \\ 1 & 830.000 & 396933.000 & 35.000 \\ 2 & 342.261 & 175626.530 & 54.000 \\ 3 & 318.000 & 120750.000 & 40.000 \\ 4 & 434.000 & 258262.000 & 77.000 \\ 5 & 619.000 & 350529.000 & 60.000 \\ 6 & 261.488 & 128939.965 & 40.000 \\ 7 & 854.000 & 426046.155 & 49.000\end{array}$

\section{SUMMARY OF INPUT TARGETS:}

$\begin{array}{cllll}\text { firm input: } & 1 & 2 & 3 & 4 \\ 1 & 12.300 & 564.000 & 28000.000 & 4.000 \\ 2 & 10.484 & 508.315 & 8068.491 & 1.387 \\ 3 & 12.000 & 603.000 & 3000.000 & 1.000 \\ 4 & 11.400 & 535.000 & 14000.000 & 2.000 \\ 5 & 11.000 & 900.000 & 20000.000 & 3.000 \\ 6 & 8.289 & 404.494 & 5609.102 & 1.024 \\ 7 & 13.612 & 764.460 & 28163.303 & 4.098\end{array}$

\section{FIRM BY FIRM RESULTS:}

Results for firm: 1

Technical efficiency $=1.000$

PROJECTION SUMMARY:

\begin{tabular}{|c|c|c|c|c|}
\hline variable & $\begin{array}{l}\text { original } \\
\text { value }\end{array}$ & $\begin{array}{l}\text { radial } \\
\text { movement }\end{array}$ & $\begin{array}{l}\text { slack } \\
\text { movement }\end{array}$ & $\begin{array}{l}\text { projected } \\
\text { value }\end{array}$ \\
\hline output 1 & 830.000 & 0.000 & 0.000 & 830.000 \\
\hline output 2 & 396933.000 & 0.000 & 0.000 & 396933.000 \\
\hline output 3 & 35.000 & 0.000 & 0.000 & 35.000 \\
\hline input 1 & 12.300 & 0.000 & 0.000 & 12.300 \\
\hline input & 564.000 & 0.000 & 0.000 & 564.000 \\
\hline input & 28000.000 & 0.000 & 0.000 & 28000.000 \\
\hline input 4 & 4.000 & 0.000 & 0.000 & 4.000 \\
\hline \multicolumn{5}{|c|}{$\begin{array}{l}\text { LISTING OF PEERS: } \\
\text { peer lambda weight } \\
11 \\
1.000\end{array}$} \\
\hline \multicolumn{5}{|c|}{$\begin{array}{l}\text { Results for firm: } 2 \\
\text { Technical efficiency }=0.807 \\
\text { PROJECTION SUMMARY: }\end{array}$} \\
\hline variable & $\begin{array}{l}\text { original } \\
\text { value }\end{array}$ & $\begin{array}{l}\text { radial } \\
\text { movement }\end{array}$ & $\begin{array}{l}\text { slack } \\
\text { movement }\end{array}$ & $\begin{array}{l}\text { projected } \\
\text { value }\end{array}$ \\
\hline output 1 & 112.000 & 0.000 & 230.261 & 342.261 \\
\hline output 2 & 23928.000 & 0.000 & 151698.530 & 175626.530 \\
\hline output 3 & 54.000 & 0.000 & 0.000 & 54.000 \\
\hline input 1 & 16.000 & -3.090 & -2.426 & 10.484 \\
\hline input & 630.000 & -121.685 & 0.000 & 508.315 \\
\hline input & 10000.000 & -1931.509 & 0.000 & 8068.491 \\
\hline input 4 & 4.000 & -0.773 & -1.841 & 1.387 \\
\hline
\end{tabular}




\section{LISTING OF PEERS:}

peer lambda weight

$\begin{array}{ll}4 & 0.489\end{array}$

$3 \quad 0.410$

Results for firm: 3

Technical efficiency $=1.000$

PROJECTION SUMMARY:

\begin{tabular}{|c|c|c|c|c|}
\hline variable & $\begin{array}{l}\text { original } \\
\text { value }\end{array}$ & $\begin{array}{l}\text { radial } \\
\text { movement }\end{array}$ & $\begin{array}{l}\text { slack } \\
\text { movement }\end{array}$ & $\begin{array}{l}\text { projected } \\
\text { value }\end{array}$ \\
\hline output 1 & 318.000 & 0.000 & 0.000 & 318.000 \\
\hline output 2 & 120750.000 & 0.000 & 0.000 & 120750.000 \\
\hline output 3 & 40.000 & 0.000 & 0.000 & 40.000 \\
\hline input 1 & 12.000 & 0.000 & 0.000 & 12.000 \\
\hline input 2 & 603.000 & 0.000 & 0.000 & 603.000 \\
\hline input & 3000.000 & 0.000 & 0.000 & 3000.000 \\
\hline input & 1.000 & 0.000 & 0.000 & 1.000 \\
\hline
\end{tabular}

LISTING OF PEERS:

peer lambda weight

$3 \quad 1.000$

Results for firm: 4

Technical efficiency $=1.000$

PROJECTION SUMMARY:

\begin{tabular}{|c|c|c|c|c|}
\hline variable & $\begin{array}{l}\text { original } \\
\text { value }\end{array}$ & $\begin{array}{l}\text { radial } \\
\text { movement }\end{array}$ & $\begin{array}{l}\text { slack } \\
\text { movement }\end{array}$ & $\begin{array}{l}\text { projected } \\
\text { value }\end{array}$ \\
\hline output 1 & 434.000 & 0.000 & 0.000 & 434.000 \\
\hline output 2 & 258262.000 & 0.000 & 0.000 & 258262.000 \\
\hline output 3 & 77.000 & 0.000 & 0.000 & 77.000 \\
\hline input 1 & 11.400 & 0.000 & 0.000 & 11.400 \\
\hline input & 535.000 & 0.000 & 0.000 & 535.000 \\
\hline input & 14000.000 & 0.000 & 0.000 & 14000.000 \\
\hline input & 2.000 & 0.000 & 0.000 & 2.000 \\
\hline
\end{tabular}

LISTING OF PEERS:

peer lambda weight

$4 \quad 1.000$

Results for firm: 5

Technical efficiency $=1.000$

PROJECTION SUMMARY:

$\begin{array}{lllll}\text { variable } & \begin{array}{l}\text { original } \\ \text { value }\end{array} & \begin{array}{l}\text { radial } \\ \text { movement }\end{array} & \begin{array}{l}\text { slack } \\ \text { movement }\end{array} & \begin{array}{l}\text { projected } \\ \text { value }\end{array} \\ \text { output 1 } & 619.000 & 0.000 & 0.000 & 619.000 \\ \text { output 2 } & 350529.000 & 0.000 & 0.000 & 350529.000 \\ \text { output 3 } & 60.000 & 0.000 & 0.000 & 60.000 \\ \text { input 1 } & 11.000 & 0.000 & 0.000 & 11.000 \\ \text { input 2 } & 900.000 & 0.000 & 0.000 & 900.000 \\ \text { input 3 } & 20000.000 & 0.000 & 0.000 & 20000.000 \\ \text { input 4 } & 3.000 & 0.000 & 0.000 & 3.000\end{array}$

LISTING OF PEERS:

peer lambda weight

51.000 
Results for firm: 6

Technical efficiency $=0.754$

PROJECTION SUMMARY:

$\begin{array}{lllll}\text { variable } & \begin{array}{l}\text { original } \\ \text { value }\end{array} & \begin{array}{l}\text { radial } \\ \text { movement }\end{array} & \begin{array}{l}\text { slack } \\ \text { movement }\end{array} & \begin{array}{l}\text { projected } \\ \text { value }\end{array} \\ \text { output 1 } & 186.000 & 0.000 & 75.488 & 261.488 \\ \text { output 2 } & 11537.000 & 0.000 & 117402.965 & 128939.965 \\ \text { output 3 } & 40.000 & 0.000 & 0.000 & 40.000 \\ \text { input 1 } & 11.000 & -2.711 & 0.000 & 8.289 \\ \text { input 2 } & 660.000 & -162.686 & -92.820 & 404.494 \\ \text { input 3 } & 7444.000 & -1834.898 & 0.000 & 5609.102 \\ \text { input 4 } & 2.000 & -0.493 & -0.483 & 1.024\end{array}$

LISTING OF PEERS:

peer lambda weight

$\begin{array}{ll}4 & 0.317 \\ 3 & 0.389\end{array}$

Results for firm: 7

Technical efficiency $=0.939$

PROJECTION SUMMARY:

$\begin{array}{lllll}\text { variable } & \begin{array}{l}\text { original } \\ \text { value }\end{array} & \begin{array}{l}\text { radial } \\ \text { movement }\end{array} & \begin{array}{l}\text { slack } \\ \text { movement }\end{array} & \begin{array}{l}\text { projected } \\ \text { value }\end{array} \\ \text { output 1 } & 854.000 & 0.000 & 0.000 & 854.000 \\ \text { output 2 } & 382933.000 & 0.000 & 43113.155 & 426046.155 \\ \text { output 3 } & 49.000 & 0.000 & 0.000 & 49.000 \\ \text { input 1 } & 14.500 & -0.888 & 0.000 & 13.612 \\ \text { input 2 } & 900.000 & -55.101 & -80.439 & 764.460 \\ \text { input 3 } & 30000.000 & -1836.697 & 0.000 & 28163.303 \\ \text { input 4 } & 6.000 & -0.367 & -1.534 & 4.098\end{array}$

LISTING OF PEERS:

peer lambda weight
$3 \quad 0.044$
10.752
$5 \quad 0.349$

\section{Dados dos autores}

Nome completo: Guilherme Bergmann Borges Vieira

Filiação institucional: Universidade de Caxias do Sul (UCS)

Departamento: Centro de Ciências Sociais (CCSO)

Função ou cargo ocupado: Professor e Pesquisador

Endereço completo para correspondência: Rua Francisco Getúlio Vargas, 1130 - CEP 95070-560 -

Caxias do Sul-RS

Telefones para contato: (54) 3218-2100

e-mail: gbbvieir@ucs.br 
Nome completo: Roberto Birch Gonçalves

Filiação institucional: Universidade de Caxias do Sul (UCS)

Departamento: Centro de Ciências Sociais (CCSO)

Função ou cargo ocupado: Professor e Pesquisador

Endereço completo para correspondência: Rua Francisco Getúlio Vargas, 1130 - CEP 95070-560 -

Caxias do Sul-RS

Telefones para contato: (54) 3218-2100

e-mail: rbgoncal@ucs.br

Nome completo: Gabriel Sperandio Milan

Filiação institucional: Universidade de Caxias do Sul

Departamento: Centro de Ciências Sociais (CCSO)

Função ou cargo ocupado: Professor e Pesquisador

Endereço completo para correspondência: Rua Francisco Getúlio Vargas, 1130 - CEP 95070-560 -

Caxias do Sul - RS

Telefones para contato: (54) 3218-2100

e-mail: gsmilan@ucs.br

Nome completo: Alexandre Querino da Rosa

Filiação institucional: Pontifícia Universidade Católica do Rio Grande do Sul (PUCRS)

Departamento: Faculdade de Administração, Contabilidade e Economia (FACE)

Função ou cargo ocupado: Aluno do Curso de Especialização em Gestão Estratégica em Logística

Endereço completo para correspondência: Rua Doutor Eduardo Chartier, 59/ ap. 205 - CEP 90520-

100 - Porto Alegre-RS

Telefones para contato: (51) 8155-0351

e-mail: alexandre.querino@yahoo.com.br

Submetido em: 09/01/2014

Aceito em: 22/10/2014 